\title{
Precise measurement of the decay $K_{L} \rightarrow \pi^{0} \gamma \gamma$
}

\author{
A. Lai, D. Marras \\ Dipartimento di Fisica dell'Università e Sezione dell'INFN di Cagliari, I-09100
} Cagliari, Italy.

A. Bevan, R.S. Dosanjh, T.J. Gershon ${ }^{1}$, B. Hay ${ }^{2}$, G.E. Kalmus ${ }^{3}$, C. Lazzeroni, D.J. Munday, M.D. Needham ${ }^{4}$, E. Olaiya, M.A. Parker, T.O. White, S.A. Wotton Cavendish Laboratory, University of Cambridge, Cambridge, CB3 OHE, U.K. ${ }^{5}$

G. Barr, G. Bocquet, A. Ceccucci, T. Cuhadar-Dönszelmann, D. Cundy, G. D'Agostini, N. Doble, V. Falaleev, L. Gatignon, A. Gonidec, B. Gorini, G. Govi, P. Grafström, W. Kubischta, A. Lacourt, M. Lenti ${ }^{6}$, S. Luitz ${ }^{7}$, I. Mikulec ${ }^{8}$, A. Norton, S. Palestini, B. Panzer-Steindel, G. Tatishvili ${ }^{9}$, H. Taureg, M. Velasco, H. Wahl CERN, CH-1211 Genève 23, Switzerland.

C. Cheshkov, P. Hristov ${ }^{2}$, V. Kekelidze, D. Madigojine, N. Molokanova, Yu. Potrebenikov, A. Zinchenko Joint Institute for Nuclear Research, Dubna, Russian Federation.

I. Knowles, V. Martin, R. Sacco, A. Walker

Department of Physics and Astronomy, University of Edinburgh, JCMB King's Buildings, Mayfield Road, Edinburgh, EH9 3JZ, U.K.

M. Contalbrigo, P. Dalpiaz, J. Duclos, P.L. Frabetti, A. Gianoli, M. Martini, F. Petrucci, M. Savrié Dipartimento di Fisica dell'Università e Sezione dell'INFN di Ferrara, I-44100 Ferrara, Italy.
A. Bizzeti ${ }^{10}$, M. Calvetti, G. Collazuol, G. Graziani, E. Iacopini, M. Veltri ${ }^{11}$ 
Dipartimento di Fisica dell'Università e Sezione dell'INFN di Firenze, I-50125 Firenze, Italy.

H.G. Becker, M. Eppard, H. Fox, K. Holtz, A. Kalter, K. Kleinknecht, U. Koch, L. Köpke, P.Lopes da Silva, P.Maruelli, I. Pellmann, A. Peters, B. Renk, S.A. Schmidt, V.Schönharting, Y. Schué, R. Wanke, A. Winhart, M. Wittgen

Institut für Physik, Universität Mainz, D-55099 Mainz, Germany ${ }^{12}$

J.C. Chollet, L. Fayard, L. Iconomidou-Fayard, J. Ocariz, G. Unal, I. Wingerter-Seez

Laboratoire de l'Accélératur Linéaire, IN2P3-CNRS,Université de Paris-Sud, 91406 Orsay, France ${ }^{13}$

G. Anzivino, P. Cenci, E. Imbergamo, P. Lubrano, A. Mestvirishvili, A. Nappi, M. Pepe, M. Piccini

Dipartimento di Fisica dell'Università e Sezione dell'INFN di Perugia, I-06100 Perugia, Italy.

R.Carosi, R.Casali, C. Cerri, M. Cirilli, F. Costantini, R. Fantechi, S. Giudici, I. Mannelli, G. Pierazzini, M. Sozzi

Dipartimento di Fisica, Scuola Normale Superiore e SezioneINFN di Pisa, I-56100 Pisa, Italy.

J.B. Cheze, J. Cogan, M. De Beer, P. Debu, A. Formica, R. Granier de Cassagnac, E. Mazzucato, B. Peyaud, R. Turlay, B. Vallage DSM/DAPNIA - CEA Saclay, F-91191 Gif-sur-Yvette, France.

M. Holder, A. Maier, M. Ziolkowski

Fachbereich Physik, Universität Siegen, D-57068 Siegen, Germany ${ }^{14}$

R. Arcidiacono, C. Biino, N. Cartiglia, M. Clemencic, F. Marchetto, E. Menichetti, N. Pastrone

Dipartimento di Fisica Sperimentale dell'Università e Sezione dell'INFN di Torino, I-10125 Torino, Italy.

J. Nassalski, E. Rondio, M. Szleper, W. Wislicki, S. Wronka 
Soltan Institute for Nuclear Studies, Laboratory for High Energy Physics, PL-00-681 Warsaw, Poland ${ }^{15}$

\title{
H. Dibon, G. Fischer, M. Jeitler, M. Markytan, G. Neuhofer, M. Pernicka, A. Taurok, L. Widhalm \\ Österreichische Akademie der Wissenschaften, Institut für Hochenergiephysik, A-1050 Wien, Austria ${ }^{16}$
}

\begin{abstract}
The decay rate of $K_{L} \rightarrow \pi^{0} \gamma \gamma$ has been measured with the NA48 detector at the CERN SPS. A total of $2558 K_{L} \rightarrow \pi^{0} \gamma \gamma$ candidates have been observed with a residual background of $3.2 \%$. The branching ratio is determined to be $(1.36 \pm$ $\left.0.03_{(\text {stat })} \pm 0.03_{(\text {syst })} \pm 0.03_{(\text {norm })}\right) \times 10^{-6}$ and the vector coupling constant $a_{v}=$ $-0.46 \pm 0.03_{(\text {stat })} \pm 0.04_{(\text {syst })}$. This result suggests that the CP-violation effects are dominating in the $K_{L} \rightarrow \pi^{0} e^{+} e^{-}$decay. An upper limit for the $K_{L} \rightarrow \pi^{0} \gamma \gamma$ decay rate in the two photon mass region $m_{\gamma \gamma}<m_{\pi^{0}}$ is also given.
\end{abstract}

1 Present address: KEK, Tsukuba, Ibaraki, 305-0801, Japan.

2 Present address: EP Division, CERN, 1211 Genève 23, Switzerland.

3 Based at Rutherford Appleton Laboratory, Chilton, Didcot, OX11 0QX, U.K.

4 Present address: NIKHEF, PO Box 41882, 1009 DB Amsterdam, The Netherlands.

5 Funded by the U.K. Particle Physics and Astronomy Research Council.

6 Present address: Sezione dell'INFN di Firenze, I-50125 Firenze, Italy.

7 Present address: SLAC, Stanford, CA 94309, USA.

8 On leave from Österreichische Akademie der Wissenschaften, Institut für Hochenergiephysik, A-1050 Wien, Austria.

9 On leave from JINR, Dubna,141980, Russian Federation

10 Dipartimento di Fisica dell'Università di Modena e Reggio Emilia, I-41100, Modena, Italy

11 Istituto di Fisica Università di Urbino, Italy

12 Funded by the German Federal Minister for Research and Technology (BMBF) under contract 7MZ18P(4)-TP2.

${ }^{13}$ Funded by Institut National de Physique des Particules et de Physique Nucléaire (IN2P3), France

14 Funded by the German Federal Minister forResearch and Technology (BMBF) under contract 056SI74.

15 Supported by the KBN grants 5P03B101120 and SPUBM/CERN/P03/DZ210/2000 and using computing resources of the Interdisciplinary Center for Mathematical and Computational Modelling, University of Warsaw.

16 Funded bythe Austrian Ministery for Traffic and Research under the contract GZ 616.360/2-IV GZ 616.363/2-VIII, and by the Fonds für Wissenschaft und Forschung FWF Nr. P08929-PHY 


\section{Introduction}

The measurement of the $K_{L} \rightarrow \pi^{0} \gamma \gamma$ decay is useful to constrain the CP conserving amplitude of the decay $K_{L} \rightarrow \pi^{0} e^{+} e^{-}$via two photon exchange. Previous measurements of the decay [1-4] have been compared with calculations performed in the framework of the Chiral Perturbation Theory $(\chi P T)$, the effective theory of the Standard Model at low energy in the hadronic sector. These predictions are best described in terms of the two amplitudes $\mathrm{A}$ and B (referring to angular momentum states $J=0$ and $J=2$ of the two photons respectively) in the Lorentz invariant expression for the double differential decay rate $[5]$ :

$$
\begin{array}{cc}
\frac{\partial^{2} \Gamma}{\partial y \partial z}=\frac{m_{K}}{2^{9} \pi^{3}}\left[z^{2} \cdot|A+B|^{2}+\left(y^{2}-y_{\max }^{2}\right)^{2} \cdot|B|^{2}\right] \\
z=\frac{\left(k_{3}+k_{4}\right)^{2}}{m_{K}^{2}}=\frac{m_{3,4}^{2}}{m_{K}^{2}} \quad y=\frac{\left|p_{K}\left(k_{3}-k_{4}\right)\right|}{m_{K}^{2}}
\end{array}
$$

with $m_{K}$ and $p_{K}$ the kaon mass and momentum, $k_{3}$ and $k_{4}$ the two photon momenta and $y_{\max }$ the kinematic bound for the $y$ variable, given by:

$$
y_{\max }=\frac{1}{2} \sqrt{\left(1-\frac{m_{\pi}^{2}}{m_{K}^{2}}\right)^{2}+\left(1+\frac{m_{\pi}^{2}}{m_{K}^{2}}\right)^{2} \times z+z^{2}}
$$

The leading $O\left(p^{4}\right) \quad \chi P T$ calculation predicts $\mathrm{B}=0$, in qualitative agreement with the experimental observation of a $z$ spectrum peaked at high values, but underestimates the $K_{L} \rightarrow \pi^{0} \gamma \gamma$ branching ratio by about a factor of three [6]. At $O\left(p^{6}\right)$ the rate and the $m_{\gamma \gamma}$ spectrum can be reproduced by adding a contribution from the VMD mechanism [5,7,8], via the coupling constant $a_{v}$ [9-11]. The VMD mechanism could enhance the state $J=2$ for the two photons, hence allowing a sizeable $\mathrm{CP}$ conserving contribution which is not helicity suppressed for the $K_{L} \rightarrow \pi^{0} e^{+} e^{-}$decay [12].

In this paper we present a new measurement of the branching ratio and decay spectrum of the decay $K_{L} \rightarrow \pi^{0} \gamma \gamma$, based on data collected in the years 1998 and 1999 with the apparatus of the NA48 experiment. Quantitative information on the VMD contribution is obtained from the spectrum at low $\mathrm{z}$ values.

\section{Experimental set-up}

The $K_{L} \rightarrow \pi^{0} \gamma \gamma$ events are collected using the NA48 detector located at the CERN-SPS and primarily devoted to measure the parameter $\epsilon^{\prime} / \epsilon$ which char- 
acterises direct CP violation in neutral Kaon decays into two pions [15][16]. Neutral Kaons are produced in interactions of $1.4 \times 10^{12} 450 \mathrm{GeV} / c$ protons on a beryllium target during 2.4 seconds every 14.4 seconds. A system of sweeping magnets and collimators defines a neutral beam of $2 \times 10^{7} K_{L}$ per burst and divergence of $\pm 0.15 \mathrm{mrad}$. A fraction of the non interacting protons are redirected by channelling in a bent crystal, to a second target, 120 metres downstream of the first, to generate a $K_{S}$ beam [17]. In order to tag $K_{S}$ decays, the protons before hitting this target are detected by an array of scintillation counters (tagger). The two beams converge with an angle of $0.6 \mathrm{mrad}$ at the calorimeter, $\approx 120$ metres downstream of the final collimators. The decay region is contained in an evacuated cylindrical vessel 89 metres long separated from the NA48 detector volume by a thin Kevlar window $0.3 \%$ of a radiation length $\left(X_{0}\right)$. The neutral beam traverses the detector inside a $16 \mathrm{~cm}$ diameter vacuum pipe. A scintillation counter (AKS) placed on the $K_{S}$ beam defines the upstream edge of the fiducial region for decays from that beam.

The most important detector element for this analysis is the quasi-homogeneous liquid Krypton electro-magnetic calorimeter ( $\mathrm{LKr}$ ) structured in 13212 readout tower cells $27 X_{0}$ deep [18]. The ionization signal from each of the cells is integrated, amplified, shaped and digitised by 10-bit FADCs at $40 \mathrm{MHz}$ sampling frequency. The energy resolution can be parameterised as:

$$
\sigma(E) / E \simeq(0.09 \pm 0.01) / E \oplus(0.032 \pm 0.002) / \sqrt{E} \oplus(0.0042 \pm 0.0005),
$$

with $\mathrm{E}$ in $\mathrm{GeV}$. The spatial and time resolutions are better than $1.3 \mathrm{~mm}$ and $300 \mathrm{ps}$, respectively, for photons with energy greater than $20 \mathrm{GeV}$.

Seven ring shaped counters (AKL), consisting of plastic scintillator and iron converters, surround the decay and spectrometer region in order to veto events with photons outside the calorimeter acceptance. Their efficiency has been estimated to be around 95\%. The charged particles are reconstructed by a magnetic spectrometer consisting of four drift chambers (DCH) and a magnetic dipole. The space resolution for each projection is $90 \mu \mathrm{m}$ and the average efficiency is better than $99.5 \%$ per plane. For this analysis the spectrometer is used to veto events with reconstructed tracks.

\section{Data taking}

Events of the $K_{L} \rightarrow \pi^{0} \gamma \gamma$ decay channel and of the channel $K_{L} \rightarrow \pi^{0} \pi^{0}$, used for normalisation, are collected by the same trigger, since their final states appear identical in all detector elements. The trigger decision is based on quantities which are derived from the sums of the energy deposited in the LKr calorimeter in groups of cells corresponding to 64 horizontal and 64 vertical slices [19]. The trigger requirements are: 
1) at maximum 5 peaks in each projection;

2) total energy larger than $50 \mathrm{GeV}$;

3) centre of gravity of the event, computed from the first moments of the energy peaks in the projections to be within $15 \mathrm{~cm}$ from the beam axis;

4) proper time of the decay, computed from the second moments, less than $5 \tau_{K_{S}}$ from the beginning of the decay region.

The trigger efficiency has been checked to be better than 99.9\% [16] using a minimum bias sample triggered by a scintillating fibre hodoscope placed in the $\mathrm{LKr}$ volume at a depth of $9.5 X_{0}$.

During the experimental runs roughly 180 Terabytes of raw data were recorded and pre-selected by an on-line software filter, whose criteria are subsequently tightened in the off-line analysis.

\section{Analysis}

The first step is to reconstruct the showers in the liquid krypton calorimeter summing the energies of the cells in a circle of $11 \mathrm{~cm}$ radius from a local maximum. The time and the position of the shower are derived from the most energetic cells and from the centre of gravity of the energy. A partial overlap between two showers is resolved using the shower profile obtained from simulation. The shower energy is corrected for:

i) the residual $0.4 \%$ non-uniformity in the calibration electronics chain, comparing energy and momentum of electrons from $K_{e_{3}}$ events as a function of the hit cell. The uniformity is improved to the $0.15 \%$ level;

ii) the $1 \%$ peak-to-peak modulation of the response as a function of the impact point in the LKr cell due to the effects of a $2 \mathrm{~mm}$ gap between electrodes in the vertical direction and to the finite integration time in the horizontal one;

iii) the energy-leakage in the beam pipe at small radius, the material before the active volume and the presence of not properly working cells;

iv) the small $(0.1 \%)$ non-linearity.

Since the $K_{L} \rightarrow \pi^{0} \gamma \gamma$ event is characterised by four clusters with the only constraint of two showers coming from a $\pi^{0}$ decay, all the events with at least four clusters are considered. Each cluster must be located well inside the calorimeter volume, i.e. within an octagon of $113 \mathrm{~cm}$ apothem, with $15 \mathrm{~cm}$ inner radius. It must be at a distance larger than $2 \mathrm{~cm}$ from any non working cell and separated from another cluster by at least $10 \mathrm{~cm}$. The cluster energy must be between $3 \mathrm{GeV}$ and $100 \mathrm{GeV}$ and the time within $3 \mathrm{~ns}$ of the event time, defined as the average of the cluster times. The total energy of the clusters must be in the $70-170 \mathrm{GeV}$ interval. 
The two photon invariant mass is:

$$
m_{\gamma_{i} \gamma_{j}}=\frac{\sqrt{E_{i} E_{j} d_{i, j}^{2}}}{z_{L K r}-z_{K}}
$$

where $E_{i}, E_{j}$ are the cluster energies, $d_{i, j}^{2}=\left[\left(x_{i}-x_{j}\right)^{2}+\left(y_{i}-y_{j}\right)^{2}\right]$ with $x_{i}$ and $y_{i}$ the horizontal and vertical positions of the i-th cluster at the LKr front face. The longitudinal coordinate of the decay vertex, $z_{K}$, is computed from the electro-magnetic calorimeter information assuming the nominal kaon mass for the parent particle:

$$
z_{K}=z_{L K r}-\frac{\sqrt{\sum_{i, j, i>j} E_{i} E_{j} d_{i, j}^{2}}}{m_{K}}
$$

where $z_{L K r}$ is the longitudinal coordinate of the LKr front face. The overall energy scale is fixed by the fit of the position of AKS counter, that vetoes all $K_{S}$ decays occurring upstream [15].

We only consider events for which there is no proton detected in the $K_{S}$ tagger in time (within $\pm 2 \mathrm{~ns}$ ) with the event; this rejects $10.65 \%$ [16] of the $K_{L}$ decays, due to accidental coincidences with a proton in the tagger.

Because of the small branching ratio, the $K_{L} \rightarrow \pi^{0} \gamma \gamma$ signal is subject to large backgrounds such as $K_{L} \rightarrow \pi^{0} \pi^{0} \pi^{0}$ decays, with missing and/or overlapping photons, mis-reconstructed $K_{L} \rightarrow \pi^{0} \pi^{0}$ decays with $\gamma$ conversion in the spectrometer and events generated by accidental pile up of particles from two different events. We distinguish two classes of cuts in the analysis: $2 \pi^{0}$ rejection and $3 \pi^{0}$ rejection.

\section{$4.12 \pi^{0}$ rejection.}

Three different combinations of $\gamma \gamma$ pairs can be formed from four showers. A $\chi^{2}$ variable is defined:

$$
\chi^{2}=\left[\frac{\left(m_{1,2}+m_{3,4}\right) / 2-m_{\pi^{0}}}{\sigma_{+}}\right]^{2}+\left[\frac{\left(m_{1,2}-m_{3,4}\right) / 2}{\sigma_{-}}\right]^{2}
$$

where $\sigma_{ \pm}$are the resolutions of $\left(m_{1,2} \pm m_{3,4}\right) / 2$ measured from the data and parametrised as a function of the lowest photon energy, typically $\sigma_{+} \approx$ $0.42 \mathrm{MeV} / c^{2}$ and $\sigma_{-} \approx 0.83 \mathrm{MeV} / c^{2}$. In order to select a $\pi^{0} \pi^{0}$ candidate the best combination is required to have $\chi^{2} \leq 13.5$, while the $\pi^{0} \gamma \gamma$ events are selected with $\chi^{2}>300$. We retain for further analysis all combinations that 
give a photon pair (that we call $m_{1,2}$ ) within $3 \mathrm{MeV} / c^{2}$ from $m_{\pi^{0}}$ and the other pair (corresponding to $m_{3,4}$ ) outside the window $110-160 \mathrm{MeV} / c^{2}$.

The $2 \pi^{0}$ background concentrates in the most interesting region for the determination of $a_{v}$, namely at low $m_{3,4}$ value $\left(m_{3,4}<240 \mathrm{MeV} / c^{2}\right)$ where the $K_{L} \rightarrow \pi^{0} \gamma \gamma$ signal is smaller. Due to mis-reconstructions one of the $\pi^{0}$ may be reconstructed with a wrong mass. This can happen in case of a $\gamma$-conversion or $\pi^{0}$ Dalitz decay with one electron outside the calorimeter acceptance that biases the photon energy reconstruction. We use the first three drift chambers as a veto against conversions and Dalitz decays if there are hits in coincidence, $\pm 20 \mathrm{~ns}$, with the event time. In addition we reject events with an overflow condition in the readout of more than one plane of the drift chambers. This condition is defined by the occurrence of more than seven hits in a DCH plane in any $100 \mathrm{~ns}$ time interval. In this case the front-end readout buffers are reset and the DCH information cannot be used reliably.

The remaining $2 \pi^{0}$ background is reduced requiring $p_{T, 4}>40 \mathrm{MeV} / c$, where $p_{T, 4}$ is the transverse momentum to the kaon direction of $\gamma_{4}$, which is the lowest-energy photon of the non- $\pi^{0}$ pair of photons. The variable $p_{T, 4}$ is on average smaller for $2 \pi^{0}$ events than for $\pi^{0} \gamma \gamma$ events and even lower when the photon energy is underestimated. Although this requirement reduces the acceptance for $\pi^{0} \gamma \gamma$ at high $y$ and low $m_{3,4}$, it does not degrade the sensitivity to the $B$ amplitude as the $\left(y^{2}-y_{\max }^{2}\right)$ factor in equation 1 is already small.

\section{2 $3 \pi^{0}$ rejection.}

In order to reduce the background from $K_{L} \rightarrow \pi^{0} \pi^{0} \pi^{0}$ decays, we require the event to have only four clusters in-time, within $3 \mathrm{~ns}$, and no activity in the AKL veto counters in coincidence with the event. As most of the $3 \pi^{0}$ background events have one or two photons outside the LKr acceptance, the reconstructed $z_{K}$ according to formula (3) moves downstream the beam axis by at least 6 metres. Therefore, the fiducial volume is restricted to the first 30 metres of the $K_{L}$ decay region. Events with missing energy are also reduced by requiring the center of gravity, computed from the first momenta of the clusters $\left(R_{C O G}\right)$, to be less than $4 \mathrm{~cm}$ from the beam axis.

The simulation of $\approx 3 \times 10^{9} K_{L} \rightarrow \pi^{0} \pi^{0} \pi^{0}$ events shows that after applying these cuts the background is composed of events with missed photons: $36 \%$ of the sample has two missing photons, while $61 \%$ of the sample results from one photon missing the detector and two showers overlapping in the LKr. The remaining events have all the six photons inside the calorimeter but only four reconstructed because of two overlaps. The corresponding showers are broader than for a single isolated photon.

Events with missed photons are rejected on the basis of a variable, $Z_{\max }$, designed to estimate, for these events, the true kaon decay vertex position. 
The same estimate, when applied to signal events, produces an unphysical result, with a distribution showing many events in the region upstream of the final collimator. The distribution of this variable is shown in figure 1 . A condition $Z_{\max } \leq-5 \mathrm{~m}$ rejects almost $99 \%$ of the $3 \pi^{0}$ background while retaining $46 \%$ of the signal.

The procedure to define $Z_{\max }$ is based on the analysis of the possible topologies of events with missed photons, as shown in the following figure:

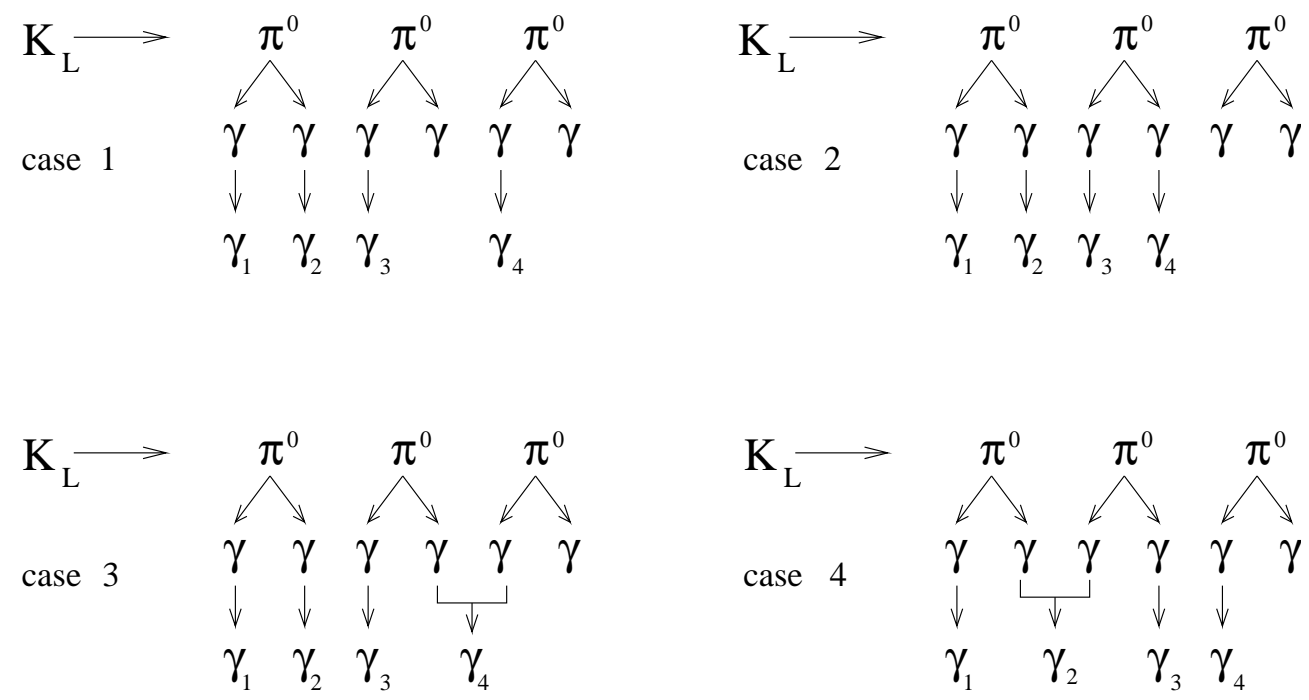

cases 1,2 and 3 :

a $\pi^{0}$ can be reconstructed and the true vertex coordinate $z_{\gamma \gamma}$ is obtained using equation (2) with $m_{\gamma_{i} \gamma_{j}}=m_{\pi^{0}}$. There are five possible $\gamma \gamma$ combinations, other than the one of the reconstructed $\pi^{0}$, but we consider only those that have $z_{\gamma \gamma}$ smaller than $\left(z_{K}-6 \mathrm{~m}\right)$. We call $Z_{\pi}$ the vertex estimate which is closest to this limit.

\section{case 4:}

one $\pi^{0}$ has a missing photon and two photons from the other $\pi^{0}$ 's overlap. Starting from the hypothesys of two $\pi^{0} \mathrm{~s}$ which share a photon, we compute, for each group of three photons, the vertex coordinate $z_{\gamma \gamma \gamma}$, assuming each photon in turn to be the superposition of two photons with the same position in the calorimeter. For example if photons 1, 2 and 3 come from two $\pi^{0} \mathrm{~s}$ and photon 2 is overlapped, then $z_{\gamma \gamma \gamma}$ is computed as:

$$
z_{\gamma \gamma \gamma}=z_{L K r}-\frac{1}{m_{\pi^{0}}}\left(\frac{1}{E_{1} E_{2} d_{1,2}^{2}}+\frac{1}{E_{2} E_{3} d_{2,3}^{2}}\right)^{-\frac{1}{2}}
$$

Out of the twelve possible combinations we consider only the triplets with an invariant mass below $\left(m_{K}-m_{\pi^{0}}\right)$ and the vertex estimate closest to, but smaller than, $\left(z_{K}-6 m\right)$.

For each event we define the variable $Z_{\max }=\max \left(Z_{\gamma \gamma}, Z_{\gamma \gamma \gamma}\right)$. The residual $3 \pi^{0}$ background originates from two overlaps of photons such that the $z_{K}$ is 


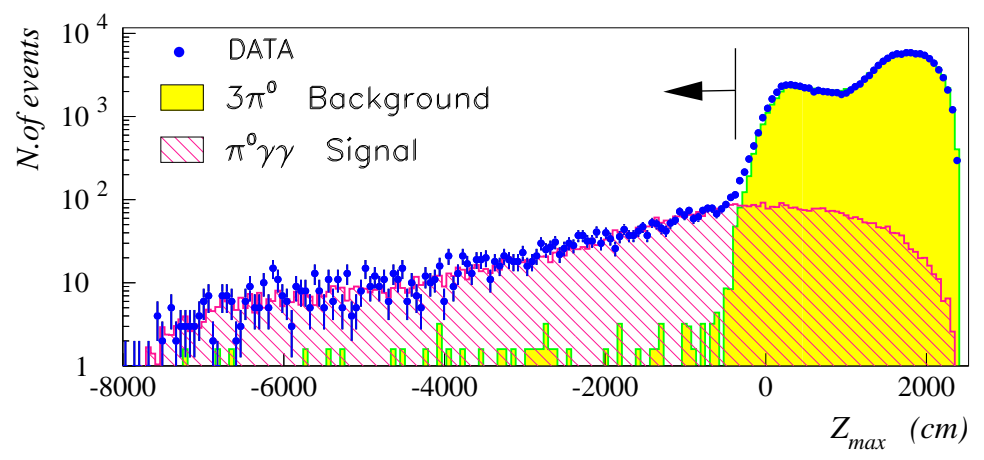

Fig. 1. Distribution of the variable $Z_{\max }$ used to reject the $3 \pi^{0}$ background with missing photons, as it looks after all the other requirements are applied. Dots are $\pi^{0} \gamma \gamma$ candidates and solid line Monte Carlo simulation. The arrow indicates the selected $Z_{\text {max }}$ region.

reconstructed unbiased, but the shape of one or two showers is different from that of a single isolated photon. As estimator of the cluster width we use the RMS of its energy profile in $x$ and $y$ :

$$
w_{x(y)}=\sqrt{\frac{\sum_{i} E_{i} \cdot \Delta x(y)_{i}^{2}}{\sum_{i} E_{i}}} \quad i \in 5 \times 5 \text { cells around the impact cell }
$$

where $\Delta x(y)_{i}$ is the distance in the $x(y)$ direction of the $i$-th cell from the center of gravity of the cluster energy. An energy-dependent cut is applied to the width of each cluster. The value of the cut is defined in terms of the average $\bar{w}$ and the standard deviation $\sigma$ of the distribution of $w$ measured in photons from good $2 \pi^{0}$. We require $w<(\bar{w}+1.8 \sigma)$ on the photons of $m_{3,4}$ and $w<(\bar{w}+3 \sigma)$ on the photons of $m_{1,2}$. The signal reduction is at the level of $15 \%$.

\section{Background evaluation}

The $K_{L} \rightarrow \pi^{0} \gamma \gamma$ candidates that survive all the above selections are 2558 in the signal region defined as $132<m_{1,2}<138 \mathrm{MeV} / c^{2}$. For 345 of these events more than one combination satisfies the $m_{1,2}$ cut and therefore the mass assignment is ambiguous.

We estimate the contamination from $2 \pi^{0}$ decays and pile-up processes directly from the data. To quantify the $K_{L} \rightarrow \pi^{0} \pi^{0} \pi^{0}$ background we rely on Monte Carlo.

We study the $K_{L} \rightarrow \pi^{0} \pi^{0}$ background in the signal region using $K_{S} \rightarrow \pi^{0} \pi^{0}$ decays from different $K_{S}$ data samples: the proton tagged $K_{S}$ sample, collected concurrently with the $K_{L}$ data, and a special $K_{S}$ high intensity run $\left(K_{S} H I\right)$ taken in 1999. In the case of the $K_{S}$ tagged sample we ask for a 
tight coincidence within \pm 0.5 ns between the tagger and the $\mathrm{LKr}$ signals. In this way we enrich the number of $2 \pi^{0}$ events contributing to the background. Conversely the only contribution to $\pi^{0} \gamma \gamma$ in this sample comes from the $K_{L}$ contamination due to accidental coincidences in the tagger, which is estimated to be $3.5 \%$ with the cut used. After applying the $\pi^{0} \gamma \gamma$ selection

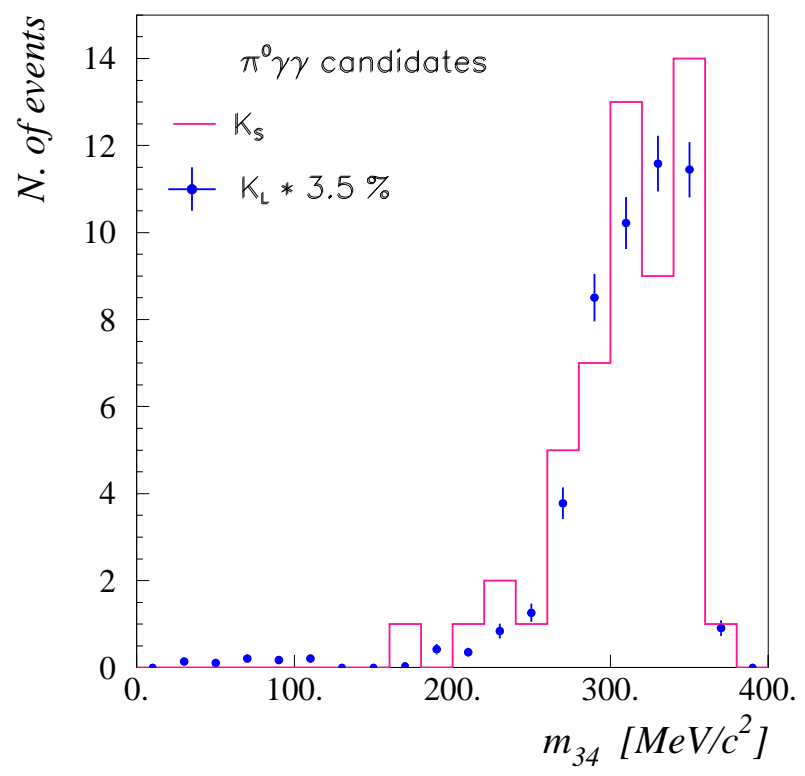

Fig. 2. $m_{3,4}$ distribution for the $3.5 \%$ of the $K_{L}$ data (dots) and for the candidates found in the $K_{S}$ tagged sample (histogram). No excess is visible in the low-mass region

criteria, the $m_{3,4}$ invariant mass distribution is shown in figure 2 for events in the tagged sample compared to the original $K_{L} \rightarrow \pi^{0} \gamma \gamma$ mass distribution scaled by the $K_{L}$ contamination factor. The $2 \pi^{0}$ background is estimated from the tiny excess of tagged events measured where the $\pi^{0} \gamma \gamma$ signal is smaller, namely for $m_{3,4}<0.240 \mathrm{MeV} / c^{2}$ while for $m_{3,4}>0.240 \mathrm{MeV} / c^{2}$ it is linearly estrapolated from control regions on the sidebands of the $\pi^{0}$ peak ( $127<m_{1,2}<130 \mathrm{MeV} / c^{2}$ or $\left.140<m_{1,2}<143 \mathrm{MeV} / c^{2}\right)$. This procedure leads to a background evaluation of $\left(4.1 \pm 1.6_{(\text {stat })} \pm 1.4_{(\text {syst })}\right)$ events. Here the systematic error is related to the subtraction of the $K_{L}$ contamination and to the fact that $K_{S}$ events have a distribution of the decay vertex populated only in the region between 6 and 18 metres.

The high intensity $K_{S}$ data sample provides about $60 \%$ of the tagged $K_{S} \rightarrow$ $\pi^{0} \pi^{0}$ statistic with negligible $K_{L}$ component. The number of background events found is in agreement with the previous estimate.

The cuts applied to reduce the $K_{L} \rightarrow \pi^{0} \pi^{0} \pi^{0}$ background are mainly of geometrical nature and can safely be simulated. The most critical cut is the one on the cluster width, for which an accurate description of the LKr response for overlapping showers is needed. For this last variable we compare data and Monte Carlo distributions in figure 3. The simulation describes the data within $3 \%$ both in shape and quantity being normalised to the Kaon flux. 


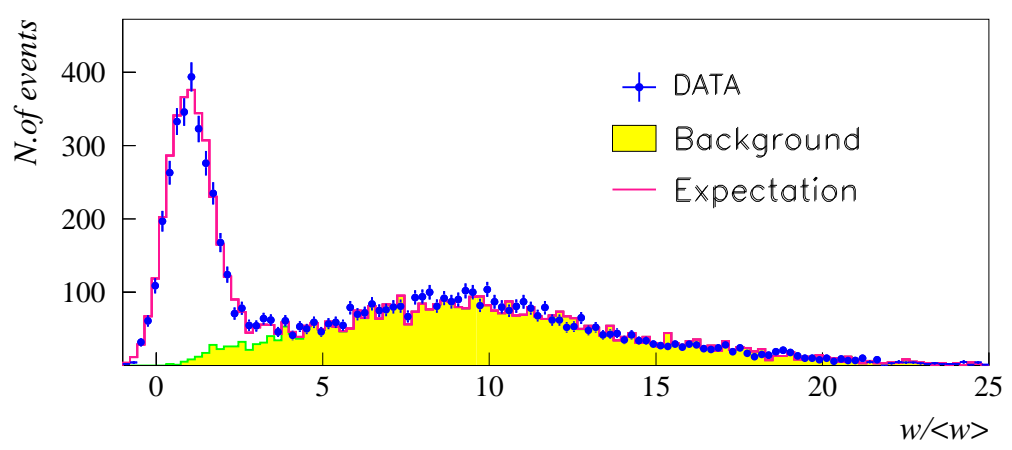

Fig. 3. Distribution of the $w$ variable used to reject the $3 \pi^{0}$ background (shaded area) with overlapped showers after all the other requirements. The white histogram corresponds to the sum of signal and background distributions. Monte Carlo data (solid line histogram) follow the experimental data (dots).

After the cut the estimated residual background is $\left(70.2 \pm 8.2_{(\text {stat })} \pm 7.0_{(\text {syst })}\right)$ events, where the systematic uncertainty comes from the non-Gaussian tail of the calorimeter response mainly due to photo-hadron reactions.

Finally, the pile-up events, which could originate for example from two overlapped in-time kaon decays, can be quantified comparing the normalised distributions of the center of gravity at the LKr for $K_{L} \rightarrow \pi^{0} \gamma \gamma$ candidates and for a good $2 \pi^{0}$ data sample. An excess at $R_{C O G}>4 \mathrm{~cm}$ is found in the $K_{L} \rightarrow \pi^{0} \gamma \gamma$ distribution, after having subtracted the $3 \pi^{0}$ background (figure 4). When the excess is linearly extrapolated under the $K_{L} \rightarrow \pi^{0} \gamma \gamma$ signal we obtain a pile-up background level of $\left(8.1 \pm 3.5_{(\text {stat }} \pm 4.0_{(\text {syst })}\right)$ events. The choice of extrapolation function is dominating the systematic error; however the linear one is expected from a uniform center of gravity distribution and is confirmed by the $R_{C O G}$ distribution for events with out-of-time clusters.

In Table 1 is reported the summary of the background evaluation in the signal region and, as cross-check, in the control regions defined above.

The overall background is $3.2 \%$. The invariant mass distributions $m_{1,2}$ and

\begin{tabular}{l|c|ccc|cc}
\hline sample & $\pi^{0} \gamma \gamma$ & $3 \pi^{0}$ & $2 \pi^{0}$ & Pile-up & Total bck & $\%$ \\
\hline signal & 2558 & $70.2 \pm 10.7$ & $4.1 \pm 2.1$ & $8.1 \pm 5.3$ & $82.4 \pm 12.1$ & 3.2 \\
sidebands & 44 & $30.6 \pm 5.9$ & $2.1 \pm 1.6$ & $4.8 \pm 3.5$ & $37.5 \pm 7.0$ & - \\
\hline
\end{tabular}

Table 1

Number of candidate and background events in the signal region $\left(132<m_{1,2}<\right.$ $\left.138 \mathrm{MeV} / \mathrm{c}^{2}\right)$ and in control regions $\left(127<m_{1,2}<130 \mathrm{MeV} / \mathrm{c}^{2}\right.$ or $140<m_{1,2}<$ $\left.143 \mathrm{MeV} / \mathrm{c}^{2}\right)$ on the sidebands of the $\pi^{0}$ peak.

$m_{3,4}$ are shown in figure 5 . The background estimate is in agreement with that found in the two control regions where 44 background events are observed in the data with an expectation of $38 \pm 7$. 

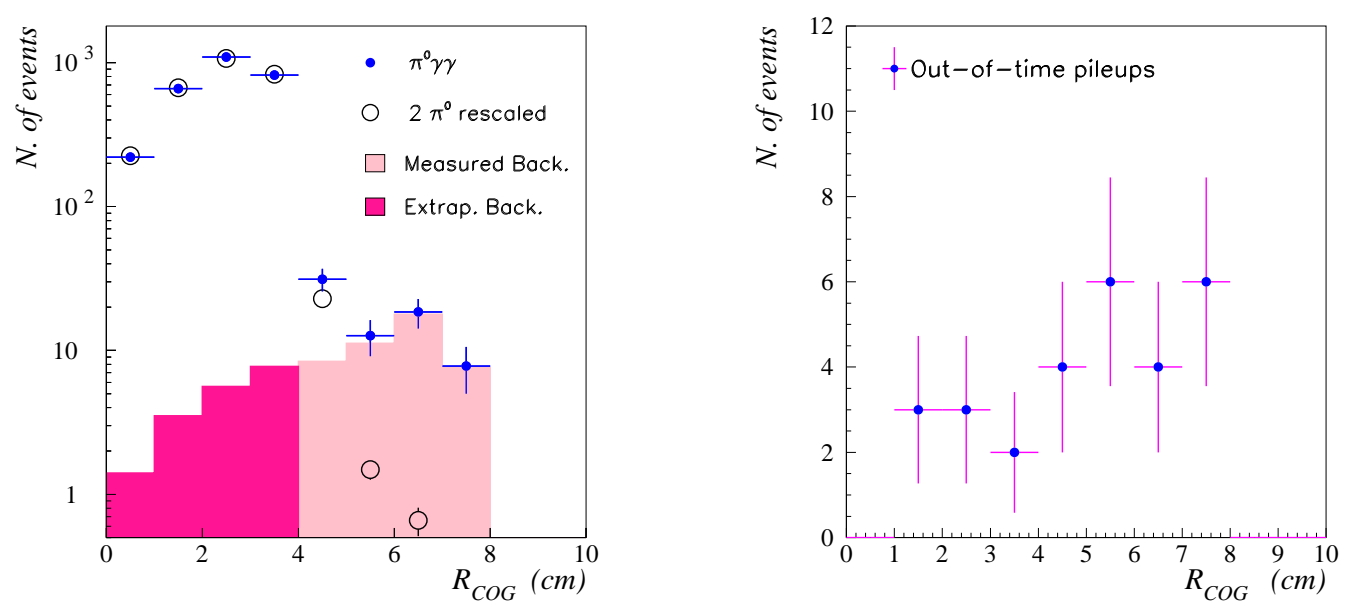

Fig. 4. $R_{C O G}$ distribution (left) for the candidate events (dots) for $2 \pi^{0}$ events (open circle). The residual background in the tail of high $R_{C O G}$ is linearly extrapolated below the peak as suggested from the $R_{C O G}$ distribution of events with out-of-time clusters (right).
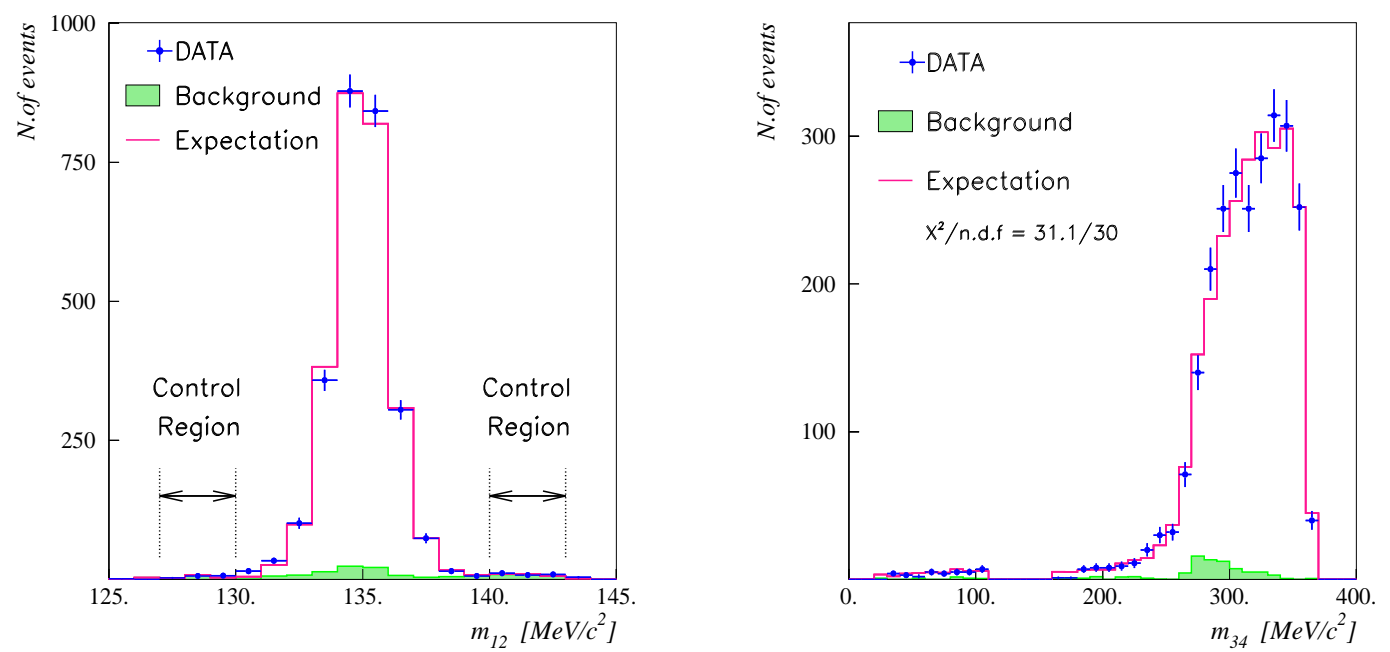

Fig. 5. $m_{1,2}$ and $m_{3,4}$ mass distributions for the $K_{L} \rightarrow \pi^{0} \gamma \gamma$ candidates events (dots) and Monte Carlo data (solid histogram) where the fitted value of $a_{v}$ has been used in the event generation. The shaded histogram is the sum of the three background types. In case of ambiguous mass assignment, only the combination with $m_{1,2}$ closest to $m_{\pi^{0}}$ mass is included in these distributions.

\section{Results and discussion}

As confirmed by the Monte Carlo, most of the events in the low $m_{3,4}$ mass region shown in figure 5 are genuine $K_{L} \rightarrow \pi^{0} \gamma \gamma$ events which suffer from the wrong combination of photons. Events with an ambiguous assignment for $m_{1,2}$ have a flat distribution in the $m_{3,4}$ mass region which will inflate the amount

of events in the low-mass $m_{3,4}<0.240 \mathrm{Mev} / c^{2}$ region. To extract a value 


\begin{tabular}{|c|c|c|c|}
\hline$m_{3,4}\left[M e V / c^{2}\right]$ & $\pi^{0} \gamma \gamma \mathrm{n}$-a events & background events & acceptance \\
\hline$[0-20]$ & 0 & 0.0 & 0.018 \\
\hline$[20-40]$ & 0 & 1.0 & 0.060 \\
\hline$[40-60]$ & 1 & 0.8 & 0.076 \\
\hline$[60-80]$ & 3 & 0.2 & 0.073 \\
\hline$[80-100]$ & 3 & 1.9 & 0.069 \\
\hline$[100-110]$ & 2 & 0.6 & 0.062 \\
\hline$[110-140]$ & 0 & 0.0 & 0.000 \\
\hline$[140-160]$ & 0 & 0.0 & 0.000 \\
\hline$[160-180]$ & 2 & 1.3 & 0.041 \\
\hline$[180-200]$ & 9 & 2.1 & 0.040 \\
\hline$[200-220]$ & 12 & 1.0 & 0.044 \\
\hline$[220-240]$ & 23 & 1.9 & 0.054 \\
\hline$[240-260]$ & 53 & 0.5 & 0.060 \\
\hline$[260-280]$ & 184 & 16.6 & 0.062 \\
\hline$[280-300]$ & 405 & 23.9 & 0.065 \\
\hline$[300-320]$ & 465 & 10.6 & 0.064 \\
\hline$[320-340]$ & 514 & 4.6 & 0.063 \\
\hline$[340-360]$ & 498 & 0.6 & 0.077 \\
\hline$[360-380]$ & 39 & 0.7 & 0.093 \\
\hline$[380-400]$ & 0 & 0.0 & 0.000 \\
\hline
\end{tabular}

Table 2

$\pi^{0} \gamma \gamma$ non-ambiguous candidates, number of background events and acceptance in 20 bins of $m_{3,4}$ in the range of $[0-400] \mathrm{MeV} / \mathrm{c}^{2}$. The normalisation factor, i.e. number of decays in the fiducial volume $N\left(K_{L}\right)$, is equal to $23.9 \times 10^{9}$. This number is based on the number of measured $N\left(K_{L} \rightarrow \pi^{0} \pi^{0}\right)$ decays.

for the vector coupling constant $a_{v}$ we exclude events with ambiguous mass assignment. The events in the low-mass region are thus reduced by $\approx 50 \%$. In Table 2 we report the numbers of $\pi^{0} \gamma \gamma$ candidate, background and acceptance in the 20 bins of the $[0-400] \mathrm{MeV} / c^{2} m_{3,4}$ interval. We perform a fit of the bidimensional distribution of the two relevant kinematic variables $m_{3,4}$ and 
$y=\left|E_{3}-E_{4}\right| / m_{K}$, defining a likelihood function as follows:

$$
\ln L=\sum_{i, N_{\text {bins }}}\left[N_{i}\left(y, m_{\gamma \gamma}\right) \cdot \ln E_{i}\left(y, m_{\gamma \gamma}\right)-E_{i}\left(y, m_{\gamma \gamma}\right)\right]
$$

where $N_{i}\left(y, m_{\gamma \gamma}\right)$ is the measured number of events in the $i$-th bin while $E_{i}\left(y, m_{\gamma \gamma}\right)$ is the corresponding expectation value given by the simulated $K_{L} \rightarrow \pi^{0} \gamma \gamma$ plus the estimated background. The model adopted for the simulation of the $K_{L} \rightarrow \pi^{0} \gamma \gamma$ process is described in [9] and it is normalised to the total number of $K_{L}$ decays in the fiducial volume. The uncertainty of the parameterisation of the $K_{L} \rightarrow 3 \pi$ vertex [13] is included in the systematic error. The best fit, with a $\chi^{2}$ of 31.1 for 30 degrees of freedom, gives:

$$
a_{v}=-0.46 \pm 0.03_{(\text {stat })} \pm 0.04_{(\text {syst })}
$$

The systematic error includes the following contributions: 0.02 from the uncertainty in the parameters of the theoretical model, 0.03 from the uncertainty of the acceptance evaluation and 0.02 from background evaluation and subtraction.

In figure 6 we show the $m_{1,2}$ distribution together with the evaluated background and the $O\left(p^{6}\right) \chi P T$ expectation for the fitted value of the vector meson exchange parameter $a_{v}$ in the three low-mass regions a) $m_{3,4} \in[30-$ 110] $\mathrm{MeV} / c^{2}$, b) $m_{3,4} \in[160-240] \mathrm{MeV} / c^{2}$ and c) $m_{3,4} \in[240-260] \mathrm{MeV} / c^{2}$. Our data clearly disfavour $a_{v}=0$.

The branching ratio is obtained from the number of $\pi^{0} \gamma \gamma$ candidates, including those with ambiguous mass assignment, normalising to the number of $\pi^{0} \pi^{0}$ events observed in the same sample of $K_{L}$ decays and selected by the same cuts, in order to minimise the uncertainty in the acceptance corrections and is found to be:

$$
B R\left(K_{L} \rightarrow \pi^{0} \gamma \gamma\right)=\left(1.36 \pm 0.03_{(\text {stat })} \pm 0.03_{(\text {syst })} \pm 0.03_{(\text {norm })}\right) \times 10^{-6}
$$

Both the correction due to the Dalitz decay difference between the $2 \pi^{0}$ normalisation mode and the $\pi^{0} \gamma \gamma$ decay and the one due to the $K_{L}-K_{S}$ interference in $\pi^{0} \pi^{0}$ decays [16] are taken into account. The uncertainty related to the experimental knowledge of the $K_{L} \rightarrow \pi^{0} \pi^{0}$ branching ratio [21] is quoted separately. The computed average value of the acceptance for the $\pi^{0} \gamma \gamma$ process is $7.62 \%$ and for the $\pi^{0} \pi^{0}$ is $8.72 \%$. The uncertainty related to this computation is the main source of the $2.5 \%$ systematic error in the branching ratio measurement since, as shown in Table 2, it has sharp variations. The residual background estimation, the uncertainty in $a_{v}$ and the exclusion of the ambiguous events each gives less than $1 \%$ variation on the branching ratio value.

As shown in figure 6 there is no evidence for a signal in the invariant mass region $m_{3,4}<m_{\pi^{0}}$ where we count $9 \pi^{0} \gamma \gamma$ candidates and $(4.5 \pm 2.7)$ background events. Using events with $y<0.2$ in 5 bins of $m_{3,4}$ between 30 and 

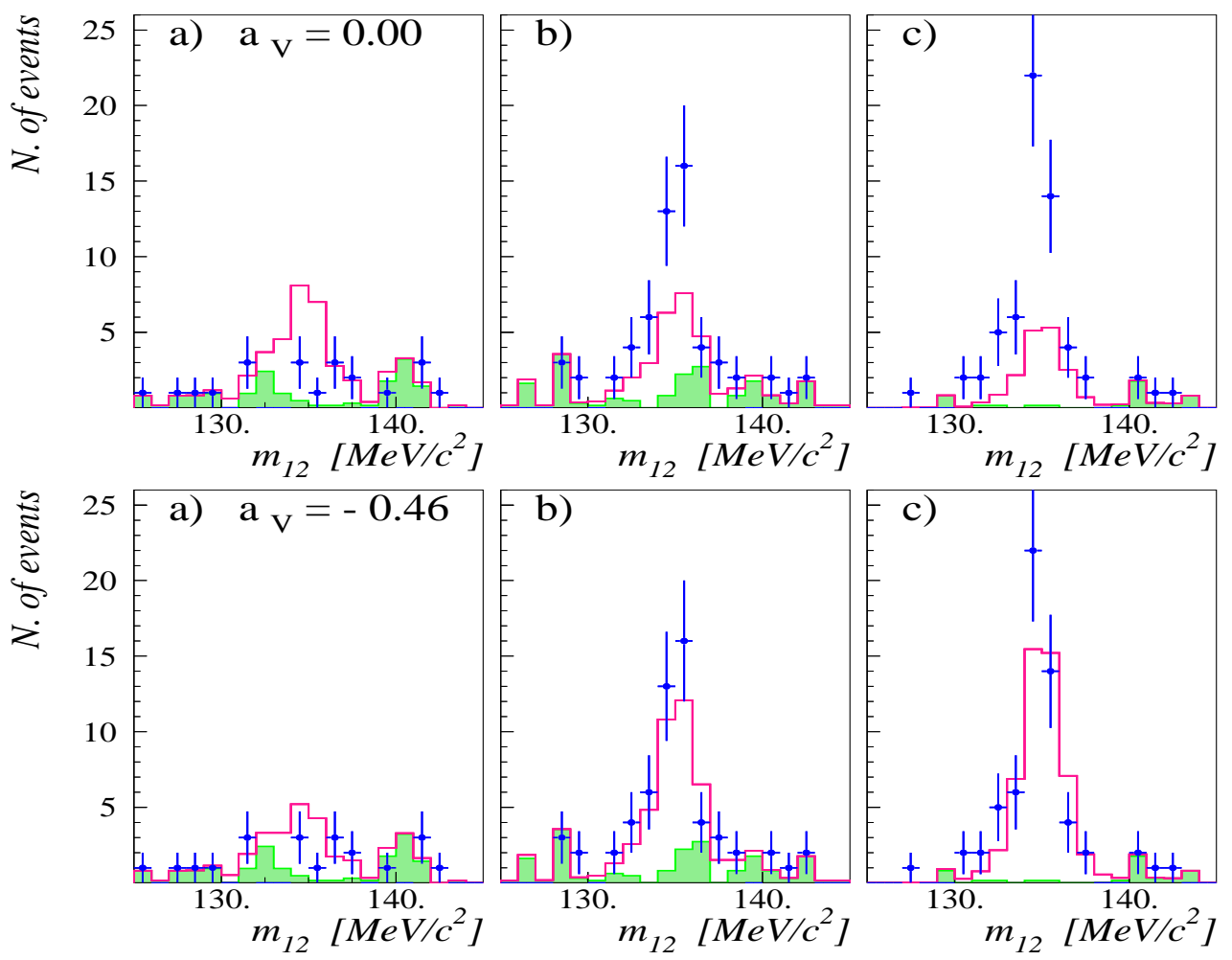

Fig. 6. Comparison between data (crosses) and the $O\left(p^{6}\right)$ expectations for $a_{v}=-0.46$ and $a_{v}=0$ (solid line histogram) in the $m_{1,2}$ variable for the three regions of the low-mass spectrum: a) $m_{3,4} \in[30-110]$, b) $m_{3,4} \in[160-240]$ and c) $m_{3,4} \in[240-260]$. Background is shown in the shaded area histogram.

$110 \mathrm{MeV} / c^{2}$, we compute a model-independent upper limit [20]:

$$
\left.B R\left(K_{L} \rightarrow \pi^{0} \gamma \gamma\right)\right|_{m_{3,4} \in[30-110] M e v / c^{2}, y \in[0-0.2]}<0.6 \times 10^{-8} \quad 90 \% \text { C.L. }
$$

For this calculation we use the acceptance computed from events generated according to phase space, in a restricted region where it is almost uniform. Given the negligible contribution of the amplitude $A(J=0)$, our measurement of the vector coupling constant $a_{v}$ allows the $\mathrm{CP}$-conserving component of the $K_{L} \rightarrow \pi^{0} e^{+} e^{-}$decay [12][14] to be predicted as:

$$
\left.B R\left(K_{L} \rightarrow \pi^{0} e^{+} e^{-}\right)\right|_{C P-\text { conserving }}=\left(4.7_{-1.8}^{+2.2}\right) \times 10^{-13}
$$

This suggests that the CP-violation effects dominate in the $K_{L} \rightarrow \pi^{0} e^{+} e^{-}$ mechanism.

\section{ACKNOWLEDGEMENT}

It is a pleasure to thank the technical staff of the participating laboratories, universities and affiliated computing centres for their efforts in the construc- 
tion of the NA48 apparatus, in the operation of the experiment, and in the processing of data. We also grateful to G.D'Ambrosio and G.Valencia for useful discussions.

\section{References}

[1] G.D. Barr et al., Phys. Lett. B 242 (1990) 523.

[2] G.D. Barr et al., Phys. Lett. B 284 (1992) 440.

[3] V. Papadimitriou et al., Phys. Rev. D 44 (1991) 573.

[4] A. Alavi-Harati et al., Phys. Rev. Lett. 83 (1999) 917.

[5] G. D'Ambrosio and J. Portoles, Nucl. Phys. B 492 (1997) 417.

[6] G. Ecker, A. Pich and E. De Rafael, Phys. Lett. B 189 (1987) 363.

[7] G. Ecker, A. Pich and E. De Rafael, Phys. Lett. B 237 (1990) 481.

[8] P. Heiliger and L.M. Sehgal, Phys. Rev. D 47 (1993) 4920.

[9] A.G. Cohen, G. Ecker and A. Pich, Phys. Lett. B 304 (1993) 347.

[10] L. Cappiello, G. D’Ambrosio and M. Miragliuolo, Phys. Lett. B 298 (1993) 423.

[11] J. Kambor and B.R. Holstein, Phys. Rev. D 49 (1994) 2346.

[12] J.F. Donoghue and F. Gabbiani, Phys. Rev. D 51 (1995) 2187.

[13] J. Kambor,J. Missimer and D. Wyler, Phys. Lett. B 261 (1991) 496.

[14] F. Gabbiani and G. Valencia, Phys. Rev. D 64, 094008 (2001)

[15] V. Fanti et al., Phys. Lett. B 465 (1999) 335.

[16] A. Lai et al., Eur. Phys. J. C 22 (2001) 231.

[17] N. Doble et al., Nucl. Instr. Meth. B 119 (1996) 181.

[18] G. Unal for the NA48 coll., Proc. 9th Int. Conf. on Calorimetry in High Energy Physics, Annecy, France, 9-14 October 2000, HEP-ex-0012011.

[19] G. Fischer et al., Nucl. Instr. Meth. A 419 (1998) 695.

[20] G.J. Feldman et al., Phys. Rev. D 57 (1998) 3873.

[21] D.E. Groom et al., Eur. Phys. J. 15 (2000) 1. 